\title{
IMAGE ENHANCEMENT USING HYBRID TRANSFORMATION TECHNIQUES
}

\author{
G.Nandini $^{1}$, P.PradeepRaju ${ }^{2}$, R.Lavanya ${ }^{3}$ \\ ${ }^{1}$ M.Tech., DECS, Elect. \& Comm. Engineering,NBKRIST, Vidyanagar \\ ${ }^{2}$ M.Tech., DECS, Elect. \& Comm. Engineering,NBKRIST, Vidyanagar \\ ${ }^{3}$ Asst. Professor, Dept. ofECE, NBKRIST, Vidyanagar
}

\begin{abstract}
Digital image processing plays a significant role in various fields through imaging techniques like image enhancement, image segmentation and image restoration etc. Image enhancement is widely used technique in some specific applications like medical image analysis and analysis of satellite images. These applications need to preserve brightness details, improvement in contrast and to highlight specific areas to clear details from noisy images. Brightness preservation enhances the visual quality of an image in a better way. Histogram equalisation is the commonly used technique for image enhancement due to its simplicity. But it shows poor performance while calculating Peak Signal to Noise Ratio(PSNR). This paper proposes a new hybrid technique using Biorthogonal wavelets and Discrete Cosine Transform with Image fusion techniques. Theresults show that this technique provides low Mean Square Error(MSE) and better PSNR value.
\end{abstract}

Keywords: Peak Signal to Noise Ratio(PSNR), Mean Square Error(MSE), image enhancement, image segmentation and image restoration

\section{INTRODUCTION}

Rapid developments in image enhancement techniques are very much useful in medical image analysis and analysis if satellite images for brightness preservation, contrast enhancement and to remove noise. Hybrid transformation techniques improve visual quality and makes further image analysis clear and easy. In low level image processing image brightness preservation is an important issue.

Image enhancement is a process of adjusting digital images to result more suitable for image display and further analysis like identifying key features and high light important areas depending on the applicationspecific.

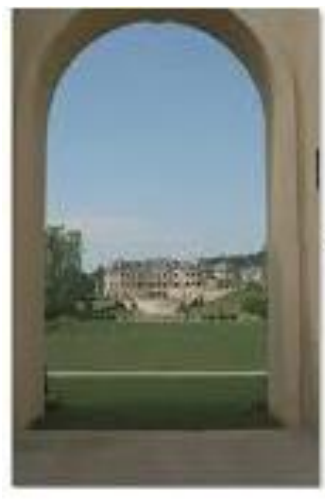

Before Enhancement
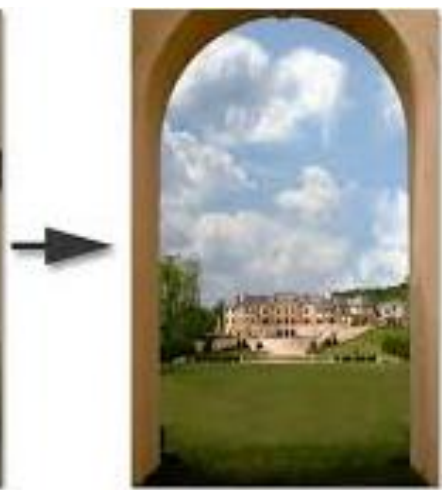

After

Enhancement
Image enhancement techniques are broadly classified into spatial \& frequency domain. Spatial domain methods are directly applicable on raw data, but frequency domain methods need transformations. Histogram processing is the simple method used to distribute grey levels of an image equally in spatial domain [1-2]. Image dependent brightness preserving histogram equalisation technique used Histogram processing for brightness preservation, and Curve let transformation for contrast enhancement is given by Mr.P. Rajvel in 2010. It shows effective results for brightness preservation but size of an image is a major constraint. This paper proposes a new hybrid transformation technique using Bi-orthogonal wavelets and discrete cosine transform with Image Fusion techniques. Results obtained through this hybrid technique shows better visual quality with low Mean Square Error (MSE) and high PSNR value for different images of variable sizes.

\section{BI-ORTHOGONAL WAVELET TRANSFORM}

Discrete Wavelet Transforms are based on small waves and became base for Multi Resolution Analysis (MRA) [4]. MRA analyses the signals (images) at different frequencies with different resolutions. The use of wavelets enables to represent the signals in frequency and time domain at the same time. And also provides the flexibility of dissolving the signal into different parts and to analyse separately. 


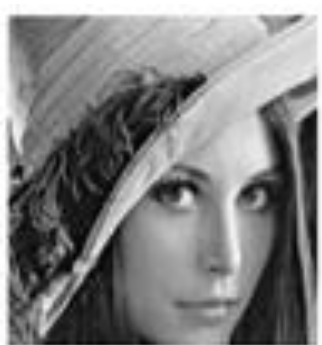

fig:a image

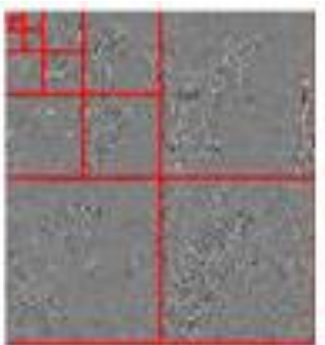

fig:b

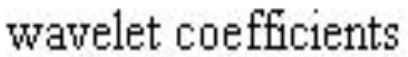

Wavelets use two functions Scaling and Transformation, Scaling captures information into different frequencies and Translation captures information into different locations [5]. $\mathrm{Bi}$-orthogonal wavelet provides perfect reconstruction by covering broader class of filters. It consists of two sets of LP Filters (for reconstruction) and HP Filters (for decomposition). In Bi-orthogonal wavelets, the decomposition and reconstruction filters are obtained from two distinct scaling functions associated with MRA's in duality. Bi-Orthogonal wavelets allows more degrees of freedom than other wavelets, provides possibility to construct symmetricwavelet function with energy compaction, which is a desirable characteristic of filter coefficients to result linear phase in transfer function. The following figure shows Decomposition \& reconstruction of filter banks for the Bi-Orthogonal case. Bi-Orthogonal scaling functions are shown below

$$
\begin{gathered}
\phi(t)=2 \sum_{n=-\infty}^{\infty} h(n) \phi(2 t-n) \\
\tilde{\phi}(t)=2 \sum_{n=-\infty}^{\infty} \tilde{h}(n) \tilde{\phi}(2 t-n)
\end{gathered}
$$

In the above equations $h(n)$ and $h^{\sim}(n)$ serve as impulse response of FIR filter $\langle\boldsymbol{\phi}(\boldsymbol{t}), \widetilde{\phi}(\boldsymbol{t}-\boldsymbol{k})\rangle=\delta(\boldsymbol{k})$ and $\left\langle\phi\left(2^{-k} t\right), \tilde{\phi}\left(2^{-k} t-n\right)\right\rangle=2^{k} \delta(n)$
The two sets of scaling functions $\varphi(t)$ and $\varphi \sim(t)$ generates subspaces.

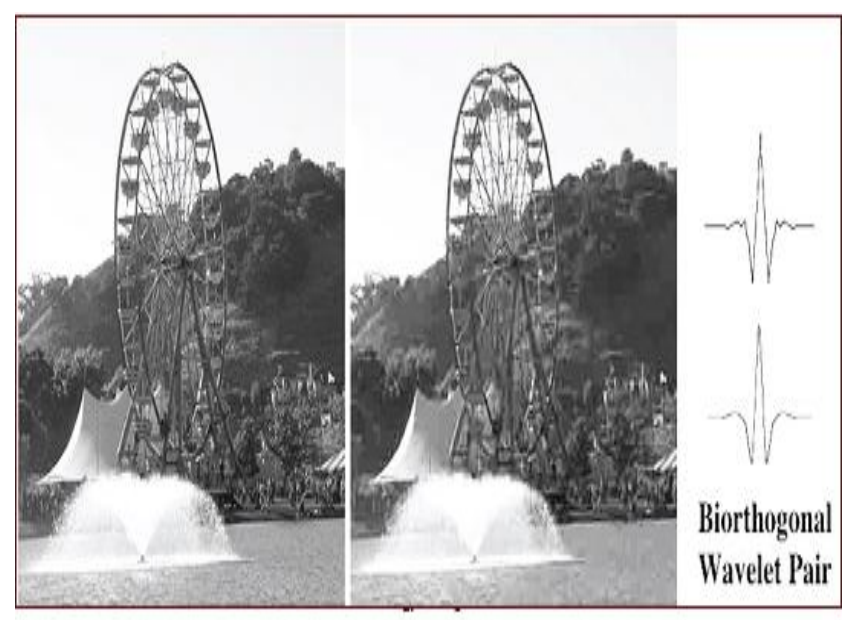

If you look closely at the top of Fermis Wheel, you will see compression "noise" on the right ingage and none on the lett

\section{2D-DISCRETE COSINE TRANSFORM}

2D Discrete Cosine Transform separates the image signal into spectral sub-bands of differing importance with respect to image's visual quality. It also transforms image from the spatial domain into the frequency domain.

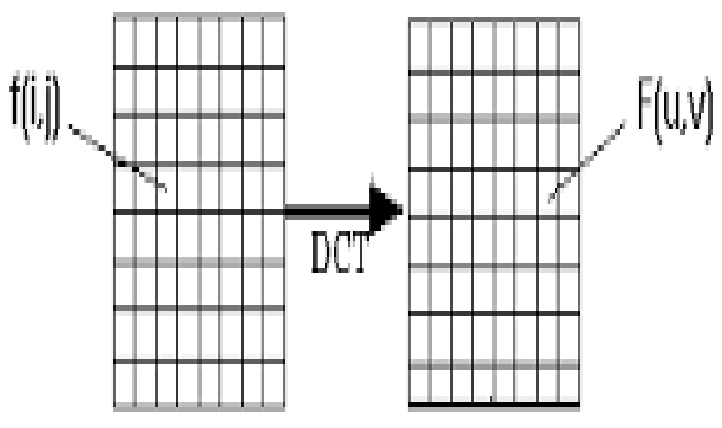

DCT is similar to the FFT but can approximate lines well with fewer coefficients. As 2D -DCT is separable, so first by applying 1D-DCT (vertically) to column's and it is again (horizontally) applied on the resultant image. The general equation is shown below.

$$
\begin{gathered}
\boldsymbol{X}_{(\mathbf{i 1}, \mathbf{i 2})}=\sum_{n 1=0}^{N 11-1} \sum_{n 2=0}^{N 2-1} x_{n 1, n 2} \operatorname{Cos}\left[\frac{\pi}{N 1}\left(n 1+\frac{1}{2}\right) i 1\right] \\
\left.\operatorname{Cos}\left[\frac{\pi}{N 2}(n 2\}+\frac{1}{2}\right) i 2\right] \ldots \ldots \ldots \ldots \ldots \ldots \ldots \ldots \ldots \ldots \ldots \ldots . . .
\end{gathered}
$$

\section{IMAGE FUSION}

Image fusion is a process of combining information from multiple images of same scene. The images may be acquired from different sensors or spatially different or of varying resolution. The main aim of image fusion techniques are to improve visual quality of images. Image Fusion can be done using various methods. Among those wavelet based methods are plays promising role to provide spatial and spectral quality and also minimises colour distortion. 


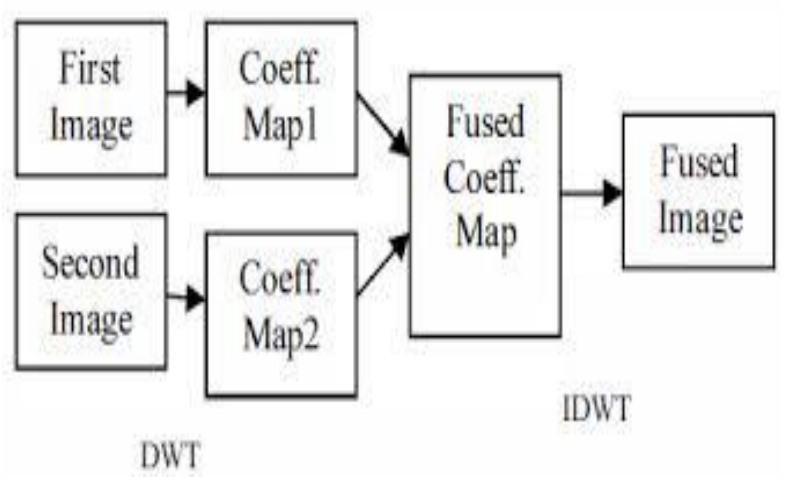

\section{PROPOSED METHOD}

Improving visual quality of images with low resolution became popular in recent research era. Individual techniques may shoe some results on visual quality, but low resolution (frequency) images need Hybrid techniques to have less Mean Square error and High Peak Signal to Noise Ratio. This proposed method involves hybrid techniques by combining various image processing techniques like DCT and BWT with image Fusion Techniques.

Basic steps followed in this algorithm are

1. Input the image (Original Image)

2. Pre-Processing (Resize Input image)

3. Apply BWT on the above image \& Save the image

4. Now apply 2D-DCT on Pre-Processed image

5. Fuse outputs of step $3 \& 4$ images using wavelets.

Performance of the above algorithm is evaluated using the metrics MSE and PSNR.

\section{RESULTS \& DISCUSSIONS}

The Proposed method is experimented in Mat lab on different images like Lena image with and without noise and on Satellite images also. Following figures shows the details.

\section{CONCLUSION}

Though Histogram processing is the simple method for brightness preservation but it is not much effective for noisy images. Higher order filters have poor time-frequency localisation. Haar wavelet is very easy to construct and recover the original image, bi-orthogonal wavelets shows perfect reconstruction with linear phase. The proposed method gives minimum MSE and maximum PSNR for low frequency images, which shows brightness levels are preserved clearly.

\section{REFERENCES}

[1]. M. J. Carlotto, "Histogram analysis using scale-space approach," IEEE Trans. Pattern Anal. Mach. Intell., vol. 9, no. 1, pp. 121-129, Jan. 1987.

[2]. M. Abdullah-Al-Wadud, Md. H. Kabir, M. A. A. Dewan, and O. Chae, "A dynamic histogram equalization for image contrast enhancement," IEEE Trans. Consumer Electron., vol. 53, no. 2, pp. 593-600, May 2007.

[3]. C.S. Burrus, R.A. Gopinath, and H. Guo, Introduction to Wavelets and Wavelet Transforms: A Primer, Prentice Hall, New Jersey, 1998.

[4]. Techniques for speckle noise removal by Jae S. Lim ; Hamid Nawab from SPIE digital library.

[5]. I. Daubechies. Ten Lectures on Wavelets, SIAM, Philadelphia, PA. 1992.

[6]. Frank W.Moore,"Evolved Multi-resolution Transformations for Optimized Image Compression and Reconstruction under Quantization", $5^{\text {th }}$ WSEAS int.conf.on wavelet Analysis and Multirate systems , Sofia, Bulgaria, October 27-29, 2005, pp.12-16.

[7]. Andreas Savakis and Richard Carbone," Discrete Wavalet Transform for core Image Processing Applications", pp.142-151, proc. Of SPIE-IS\&T Electronic Imaging, SPIE Vol.5761, 2005.

[8]. Javed Akthar et al,'Image Compression with Different types of Wavelets", ICET'06, IEEE 2006, pp.133-137.

[9]. N.B.Chopade et al," Analysis of Discerete Wavelet Based Image Compression Technique: A Review", Journal of Scientific and Industrial Research, Vol.68, Nov'09, pp.915-919.

[10]. Gerlind Plonka et al," A New Hybrid Method for Image Approximation using the easy path Wavelet Transform", IEEE Transaction on Image Processing, Vol.20, No-2, February 2011, IEEE 2011. Pp.372-381.

[11]. Stephane G.Mallet (1989)," A Theory for Multiresolution Signal Decomposition: The Wavelet Representation", IEEE Transactions on Pattern Analysis and Machine Intelligence, pp.674-693.

[12]. M. Antonini, M. Barlaud, P. Mathieu, and I. Daubechies, "Image coding using

the wavelet transform," IEEE Tran. on Image Processing, vol. 1, no. 2, Apr.1992, pp.205-220.

[13]. Shen, Gilbert G. (2001). Wavelets and Other Orthogonal Systems. Boca Raton: Chapman. ISBN 1584882271 .

[14]. Javed Akhtar et al, "Image Compression with Different Types of Wavelets”, ICET'06, IEEE 2006, pp.133-137.

[15]. T. Acharya and P. S. Tsai, JPEG2000 Standard for Image Compression: Concepts, Algorithms and VLSI Architectures. John Wiley \& Sons, Hoboken, New Jersey, 2004.

\section{BIOGRAPHIES}

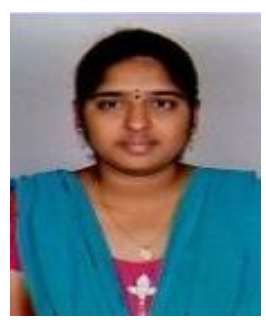

G.Nandini pursuing M.Tech degree in Digital Electronics and Communication Systems (DECS) from NBKRIST, Vidyanagar. Received B.Tech Degree in Electronics and Communication Engineering from Gokula Krishna College of Engineering, Sullurpet in 2014.

Email: nandini02nandu@gmail.com 


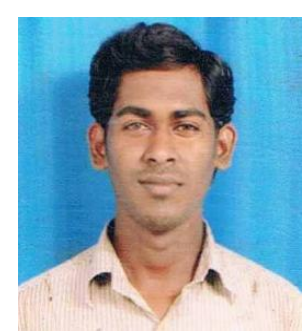

P.Pradeep Raju persuing M.Tech degree in Digital Electronics and Communication Systems (DECS) from NBKRIST, Vidyanagar. Received B.Tech Degree in Electronics and Communication Engineering from Priyadarshini college of engineering, Sullurpet in 2014.

Email: Pradeepraju475@gmail.com

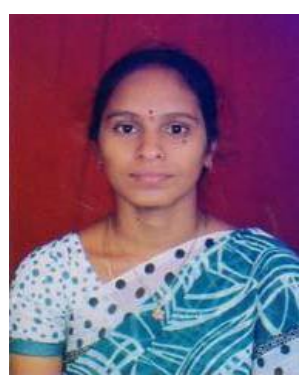

R.Lavanya working as Asst. Prof. in NBKRIST, Vidyanagar. Persuing Ph.D in KL University. Received M.Tech the degree in Digital Electronics and Communication Systems (DECS) from JNTU Anantapur and B.Tech degree in Electronics and Communication Engineering from Narayana Engineering College, Gudur.

Email: lavanyaravala@gmail.com 